\title{
Dynamic Matching and Bargaining with Heterogeneous Deadlines.*
}

\author{
Sjaak Hurkens ${ }^{\dagger} \quad$ Nir Vulkan ${ }^{\ddagger}$
}

August 4, 2014

\begin{abstract}
This paper analyzes bargaining outcomes when agents do not have stationary time preferences (as represented by a constant discount factor) but are pressed by firm deadlines. We consider a dynamic model where traders with heterogeneous deadlines are matched randomly into pairs who then bargain about the division of a fixed surplus. A trader leaves the market when an agreement has been reached or when his deadline expires. Our analysis encompasses both the case of perfect and imperfect information about the partner's deadline. We define, characterize and show the existence of a stationary equilibrium configuration. We characterize when delay occurs and when deadlines are missed in equilibrium and show that the payoffs of traders are strictly increasing and concave in own deadline, unless bargaining takes place under imperfect information and no delay occurs, in which case all pairs immediately agree on an almost even split. We provide comparative statics exercises and illustrate our results by some examples.
\end{abstract}

Key Words: Bargaining, deadlines, markets.

JEL Classification Numbers: C73; C78.

\footnotetext{
*This paper replaces and joins two earlier papers by the same authors, namely "Dynamic Matching and Bargaining: The Role of Private Deadlines" and "Dynamic Matching and Bargaining with Heterogeneous Deadlines". Hurkens gratefully acknowledges financial support from the Spanish Ministry of Economy and Competitiveness, through grant ECO2012-37065, from AGAUR through grant 2014SGR510 and through the Severo Ochoa Programme for Centres of Excellence in R\&D (SEV-2011-0075). We thank the editor, two anonymous referees, Yossi Feinberg, Zvika Neeman, Larry Samuelson and various seminar audiences for helpful comments.

${ }^{\dagger}$ Institut d'Anàlisi Econòmica (CSIC) and Barcelona GSE, Campus UAB, 08193 Bellaterra, Spain. E-mail: sjaak.hurkens@iae.csic.es.

${ }^{\ddagger}$ Saïd Business School and Worcester College, University of Oxford, Park End Street, Oxford OX1 1HP, United Kingdom. E-mail: Nir.Vulkan@sbs.ox.ac.uk.
} 


\section{Introduction}

The driving force of any dynamic bargaining model is the assumption that people prefer to realize gains early rather than late. If bargaining partners do not care about the time of agreement, there is no incentive to come to agreements in the first place and bargaining could go on forever. The eagerness to reach early agreements has traditionally been modelled by making the (expected) bargaining surplus shrink over time. This can be done by introducing a discount factor strictly less than one, by assuming a positive probability of breakdown of negotiation, or by assuming that bargaining partners face a fixed cost of bargaining per period. All these models of time preferences assume stationarity (Rubinstein, 1982): agreement $x$ today is preferred over agreement $y$ tomorrow if and only if agreement $x$ in period $t$ is preferred over agreement $y$ in period $t+1$. This assumption implies that, in the subgame perfect equilibrium of the infinite alternating offer game, it does not matter how much time has elapsed because the bargaining cost is sunk. In this paper, we want to consider deadlines as an alternative way to express a preference for early agreements in bargaining. In our interpretation, an agreement $x$ reached after the deadline has expired has no value, whereas it has a positive value as long as it is reached before the deadline. This violates the stationarity assumption.

Deadlines are present in many real bargaining situations and one would like to know how deadlines influence the bargaining strategies and outcomes. In particular, we will be interested in the case that the bargaining partners may have different deadlines and will investigate how a particular agent's bargaining behavior changes over time as his deadline comes closer. This is especially relevant when bargaining is between parties who have the opportunity to start negotiating with alternative partners. For example, in the real-estate market a house owner and a potential buyer may negotiate over the price, but both parties can break off the negotiation and start bargaining with alternative potential buyers or sellers. In financial over-the-counter markets government, municipal, and corporate bonds, bank loans, and derivatives are traded through bilateral bargaining. ${ }^{1}$ The possibility to break off negotiations becomes increasingly important when there are many potential sellers and buyers who can contact each other without too much friction. Our work is therefore also motivated by the large growth of person-to-person trade facilitated by the internet.

We have several motives for considering deadlines as an alternative way of modelling time

\footnotetext{
${ }^{1}$ See, e.g., Duffie et al. (2005) and Vayanos and Wang (2007).
} 
preferences. First, deadlines are easy to understand. It is presumably easier for a person to state by which date an agreement must be reached (say a month from now) than to make precise how much he is willing to pay extra to have an agreement today rather than tomorrow, which is basically what one needs to do in the case of standard discounting. Unemployment benefits are temporary and a job searcher's reservation wage will thus depend on how long he is still entitled to it. Also, when people negotiate on behalf of a principal ${ }^{2}$ or a constituency ${ }^{3}$ they are often given a firm deadline. Second, software agents that bargain on behalf of people using the internet must often be programmed with a deadline in order to ensure the termination of the protocol in which they take part. ${ }^{4}$ Finally, Merlo and Ortalo-Magné (2004) suggest that deadlines of sellers may explain their empirical observation that list prices of real estate decline over time, whereas other existing theories cannot explain that. Moreover, they observe that a significant fraction of sellers who initially reject offers end up accepting a lower offer. We will show that the existence of a deadline can indeed account for both observations.

This paper studies the interaction of large groups of buyers and sellers who arrive at an exogenous rate to the market. Buyers and sellers are randomly matched into pairs and bargaining takes place in each match about the division of the surplus. We assume that the size of the surplus is fixed in order to focus on the effect of heterogeneous deadlines. Bargaining is modeled by randomly picking one of the two traders in the pair and let him make a take it or leave it offer. If an agreement is reached, the traders disappear with their gains from the market. If there is no agreement, and a trader's deadline has expired, the trader will disappear from the market with no surplus. In the case of disagreement and a non-expiring deadline, the trader returns next period in which he will again be matched, with a different partner. The deadline of this trader has then been reduced by one. A trader with deadline $i$ has in total $i$ opportunities to come to an agreement with his assigned partner. After $i$ disagreements the trader receives zero surplus and disappears from the market. We consider separately the cases where trading partners do and do not observe each other's deadline. Presumably, depending on the application at hand, either case may be the relevant one. Our analysis allows for some comparisons.

Although entry is assumed to be exogenous and constant over time, the total mass of buyers and sellers present in the market may change over time because exit is endogenous. On top of that, the proportion of traders with short deadlines may change over time if traders with

\footnotetext{
${ }^{2}$ See for example, Burtraw (1992), Segendorff (1998) and Bester and Sákovics (2001).

${ }^{3}$ See Cai (2000).

${ }^{4}$ For example, Jennings et al. (1996) detail the application of ADEPT (Advanced Decision Environment for Process Tasks) agents in British Telecom's customer quote business process. Chavez and Maes (1996) consider MIT's Kasbah experiment (where agents bought and sold goods on behalf of people). In both cases deadlines were central to the design of the bargaining agents. See also Sandholm and Vulkan (2000) for a general discussion of the role of deadlines in e-commerce applications using software agents.
} 
short deadlines are more likely to come to agreements then traders with long deadlines, because we do not assume that exiting traders are replaced by new traders of the same type. We will be interested in the stationary state of the model, where the total mass of traders and the relative frequencies of deadlines remain constant over time. This allows us to focus on how the distribution of deadlines of the new traders flowing into the market affects the outcome of bargaining, payoffs and the possible existence and length of delays.

We define and show the existence of a stationary equilibrium, both for the case of perfect and imperfect information about the partner's deadline. In the case of perfect information we show that the equilibrium payoff is strictly increasing and strictly concave in deadline. We show that it is possible that when traders with relatively long deadlines are matched they choose, in equilibrium, not to trade and go back to the market in the next period. Matches in which at least one trader has a short deadline end in agreement, therefore deadlines are never missed. We characterize, in terms of the discount factor and the distribution of deadlines of traders who flow into the market in every period, whether and when delay will occur. We examine the comparative statics of our analysis and show that delay can occur frequently and can have a large negative effect on welfare. For the case of imperfect information about the partner's deadline, we show that in an equilibrium without delay all traders achieve the same expected payoff. Such an equilibrium exists if and only if the probability of meeting a trader with deadline 1 is below some threshold. It does not depend on the relative frequency of higher deadlines. Hence, in general one cannot say that delay is more likely under perfect or imperfect information. In an equilibrium with delay expected payoffs are strictly increasing in deadline. Contrary to the case of perfect information, deadlines may be missed altogether which then results in a large welfare loss. Imperfect information about deadlines thus sometimes leads to a more efficient outcome than perfect information, but it can also cause large welfare losses.

It is worth emphasizing that delay may exist even when there is perfect information in our model, immediate agreement is always efficient, and there are no transaction or switching costs. Most models of bargaining aimed at explaining the existence of delay are either based on the assumption of imperfect information so that the passing of time can signal relevant information about valuations, or they employ the multiplicity of equilibria to construct credible threats. ${ }^{5}$ Search and matching models that explain delay and inefficiencies rely heavily on the presence of positive transaction or switching cost. When frictions disappear, the search and bargaining

\footnotetext{
${ }^{5}$ See Sobel and Takahashi (1983) and Admati and Perry (1987) for examples of delay through signalling and Haller and Holden (1990) and Sákovics (1993) for examples of delay through threats using multiplicity of equilibria. Exceptions are, among others, Merlo and Wilson (1995) in an environment where the bargaining set changes over time in a stochastic manner and Fershtman and Seidmann (1993) who allow for endogenous commitments to not accept proposals that are worse than previously rejected proposals.
} 
outcome usually converges to the competitive outcome. ${ }^{6}$

The rest of the paper is organized in the following way: Section 2 presents the model. In section 3 we define and show existence of stationary equilibrium configurations and analyze their properties under the assumption that bargaining takes places under perfect information about deadlines. We also discuss how the model and results are extended to the case where the numbers of buyers and sellers, as well as the distributions of deadlines of buyers and sellers are asymmetric. In section 4 we do a similar exercise under the assumption that bargaining takes place under imperfect and private information. We discuss related literature in section 5. Section 6 concludes. Proofs are collected in the Appendix.

\section{The Model}

We consider a model with a continuum of sellers and buyers (of mass 1 each $^{7}$ ) flowing into the market every period. All sellers have one unit of a good they produced at zero cost and all buyers have unitary demands for this good, which they all value at one. The only difference between different traders is their deadline. The deadline of a trader is an integer number from $\{1,2, \ldots, N\}$ that indicates how many periods are remaining for this trader to conclude a deal. If a trader fails to conclude a deal at the last opportunity he misses his deadline and his utility is zero. That is, a trader with deadline 1 will have to make a deal immediately or his opportunity will be lost. Such a trader will accept any deal that gives him a positive utility. On the other hand, traders with a long deadline will be able and willing to reject certain deals and wait for better opportunities in the future.

We assume that proportion $p_{i}$ of the sellers (buyers) that flow into the market place every period has deadline $i$. The procedure for closing trades is as follows: in each period $t \in \mathbb{Z}$ each buyer is matched with a seller. One trader in each pair is chosen at random and becomes the proposer (with probability one half). This trader makes a proposal which can be accepted or rejected. In the first case trade takes place and traders disappear from the market. In the second case no trade takes place and both traders go back to the market and become matched next period (with different partners), as long as their deadline has not expired. Of course, their deadline will then be reduced by one.

We will be interested in the steady state or stationary equilibrium, which will be defined formally below. A stationary equilibrium is an equilibrium where all buyers (sellers) with the

\footnotetext{
${ }^{6}$ For exceptions, see Serrano (2002) and Shneyerov and Wong (2010a).

${ }^{7}$ Our model and results are readily extended to allow for unequal numbers of buyers and sellers, and also for asymmetric distributions of deadlines for buyers and sellers. In order to save on notation and to improve the exposition, we postpone discussion of the more general model to section 3.5 and confine ourselves here to the symmetric case.
} 
same deadline make and accept the same proposals (independent of the time period $t$ ) and where the mass of traders in the market place and the distribution of deadlines among the buyers (sellers) (denoted by $q$ ) remains constant over time. There are two different equilibria possible. In the first, which we will refer to as the no delay case, trade occurs in each matching. In this case the stationary distribution $q$ of deadline types is simply given by $p$. In the second, which we will refer to as the delay case, there is no trade taking place in some matches. In this case the stationary distribution $q$ will be different from the inflow distribution $p$.

We will assume that traders discount late trades by a factor $\delta \leq 1$. It will become clear later on that the role of the discount factor is not as important as in standard bargaining models. The reason is that traders with longer deadlines will close better deals than traders with shorter ones. This gives traders an incentive to make deals early, even when the discount rate is equal to one. However, if we do not discount utilities, there is no cost of having delay, as long as deadlines are never missed.

\section{Equilibrium Analysis under Perfect Information about Dead- lines}

In this section we will assume that the traders within a match learn each other's deadline before bargaining starts. A pure strategy for a trader thus must specify the offers he makes when chosen as a proposer and the offers he accepts as a responder, both as a function of his own deadline, as well as of his trading partner's deadline. We will restrict our attention, as is quite standard in the search and matching literature, to stationary equilibrium, which simplifies the analysis. ${ }^{8}$ In a stationary equilibrium the mass of traders and the distribution of deadlines remains constant over time. Moreover, strategies are time and history independent.

Given the assumed symmetry between both sides of the market, we do not need to distinguish between sellers and buyers and will just refer to both as traders. A stationary equilibrium is to a large extent characterized by the expected equilibrium payoff $w_{i}$ of a trader with deadline $i=1, \ldots, N$, because these equilibrium payoffs determine reservation values for responders which in turn determine optimal proposal strategies. ${ }^{9}$ That is, responder $j$ will accept offers strictly above and reject offers strictly below $\delta w_{j-1}$. Moreover, proposer $i$ will offer responder $j$ exactly $\delta w_{j-1}$ if he prefers this offer to be accepted (and receiving $1-\delta w_{j-1}$ ) over getting $w_{i-1}$ next period. Only when $\delta\left(w_{i-1}+w_{j-1}\right)=1$, it is not obvious whether agreement between traders $i$

\footnotetext{
${ }^{8}$ There are some exceptions though. Manea (2012) analyzes matching and bargaining models where inflow distributions are not necessarily stationary.

${ }^{9}$ For convenience we denote $w_{0}=0$.
} 
and $j$ will obtain or not. The probability of agreement will have to be explicitly specified because it will feed back into the (stationary) distribution and it cannot be deduced from equilibrium payoffs only.

To be more precise, if there is a strictly positive surplus to divide (that is, when $\delta\left(w_{i-1}+\right.$ $\left.w_{j-1}\right)<1$, agreement will be reached for sure and the proposer will extract the full surplus. Namely, a responder with deadline $j$ must accept any proposal that gives her strictly more than $\delta w_{j-1}$. In equilibrium she must then also accept a proposal of exactly $\delta w_{j-1}$ with probability 1 , because the proposer can guarantee acceptance with probability one by just offering slightly more to the responder.

If there is no mutually beneficial agreement possible (that is, when $\delta\left(w_{i-1}+w_{j-1}\right)>1$ ), no agreement will result. Either the responder is offered strictly less than $\delta w_{j-1}$ (which is of course rejected) or she is offered exactly this amount, but she still rejects it with probability equal to $1 .{ }^{10}$

Finally, in the knife-edge case that $\delta\left(w_{i-1}+w_{j-1}\right)=1$ there is of course only one possible agreement. Such an agreement must give both parties exactly their disagreement payoff. Both parties will be indifferent as to whether such a proposal would be accepted or not. In particular, the proposer is not willing to offer anything more to the responder, so he cannot guarantee acceptance. What is important for the equilibrium outcome is the probability that agreement will take place in this case, because it influences the stationary distribution of types. We will denote this probability by $A_{i j} .{ }^{11}$

\subsection{Definition and existence of equilibrium}

As explained before, a strategy profile is characterized by a vector of expected payoffs $w=$ $\left(w_{1}, \ldots, w_{N}\right)$ and a matrix $A$ of acceptance probabilities. The interpretation is that a proposer with deadline $i$ offers $\delta w_{j-1}$ to a responder with deadline $j$, and this responder accepts this proposal with probability $A_{i j}$. (And he would accept any better proposal and reject any worse proposal with certainty.) It will be convenient to explicitly include in our formal definition of a stationary subgame perfect equilibrium configuration ${ }^{12}$ the vector $z=\left(z_{1}, \ldots, z_{N}\right)$, where $z_{i}$ denotes the mass of traders with deadline $i$.

Definition 1 A triple $(z, w, A)$ is a stationary subgame perfect equilibrium configuration if the

\footnotetext{
${ }^{10}$ The responder would be indifferent between accepting and rejecting but the proposer would not be willing to make that proposal if he expected it to be accepted with positive probability.

${ }^{11}$ One should realize that payoffs arise endogenous in this model and one cannot rely on generic inflow distributions or discount factors to avoid this case of double indifference.

${ }^{12}$ We do not use the term stationary subgame perfect equilibrium, as that would implicitly refer to strategies. As argued above, the strategies cannot always be pinned down exactly.
} 
following conditions hold:

Acceptance Probabilities $A$ is an $N \times N$ symmetric matrix with $A_{i j}=1$ if $\delta\left(w_{i-1}+w_{j-1}\right)<$ $1, A_{i j}=0$ if $\delta\left(w_{i-1}+w_{j-1}\right)>1$ and $A_{i j} \in[0,1]$ otherwise.

Stationarity $z_{N}=p_{N} ; z_{i}=p_{i}+z_{i+1}\left(\sum_{j} q_{j}\left(1-A_{i+1 j}\right)\right)$ where $q_{j}=z_{j} / \sum_{k=1}^{N} z_{k}$

Expected Payoffs $w_{i}=\frac{1}{2} \delta w_{i-1}+\frac{1}{2}\left(\sum_{j=1}^{N} q_{j}\left[A_{i j}\left(1-\delta w_{j-1}\right)+\left(1-A_{i j}\right) \delta w_{i-1}\right]\right)$ for all $i$.

The first condition simply reflects our previous discussion about whether agreement will take place or not. Only in the case that both traders in the match are indifferent with respect to acceptance, we allow for interior probabilities of acceptance. The stationarity condition simply says that, when traders follow the strategies given by $(w, A)$, then the mass of traders with deadline $i$ will remain constant at $z_{i}$. Namely, it will be equal to the sum of new traders with deadline $i$ and old traders with deadline $i+1$ who did not come to an agreement. Finally, the last condition describes the relation between the expected equilibrium payoffs for traders with different deadlines, by linking bargaining outcomes with the endogenous disagreement points: A responder with deadline $i$ obtains always $\delta w_{i-1}$ (either because that is what is exactly offered and accepted or because an unacceptable offer is refused). A proposer with deadline $i$ will obtain $1-\delta w_{j-1}$ if the proposal is accepted and $\delta w_{i-1}$ otherwise.

We first establish the existence of a stationary subgame perfect equilibrium configuration.

Proposition 2 For any inflow distribution $p$ and any discount factor $\delta \in(0,1]$ there exists a stationary subgame perfect equilibrium configuration.

\subsection{Properties of the equilibrium}

Proposition 3 In any stationary subgame perfect equilibrium configuration expected payoffs are strictly increasing and strictly concave in the deadline.

An immediate implication of the fact that equilibrium payoffs are strictly increasing in deadline is that the probability of proposals being accepted is weakly decreasing both in the proposer's and in the responder's deadline. In particular, increasing the deadline of both traders by one (and thus increasing the average deadline by 1 , leaving the absolute difference between the deadlines the same) will weakly reduce the probability of agreement. The next Corollary obtains a much stronger result and reveals thus a bit more about the structure of equilibria, by using the fact that equilibrium payoffs are strictly concave in deadline. Namely, it states that the probability of agreement is weakly increasing in the absolute difference between the two deadlines, when keeping the average of the deadlines constant. So, for example, if the pair of traders with 
deadlines $(3,4)$ agrees, then the pair of traders with deadlines $(2,5)$ must agree as well. And if the pair of traders $(3,5)$ does not agree, then the pair of traders $(4,4)$ does not either. Recall that $A$ is symmetric and therefore it is enough to consider entries $A_{i j}$ on or above the diagonal.

Corollary 4 Let $\delta<1$ and let $(z, w, A)$ be a stationary subgame perfect equilibrium configuration. Then $A_{1 j}=A_{i 1}=1$ for all $i$ and $j$. Also, $A_{22}=1$. Moreover, for any two pairs of traders $(i, j)$, with $1<i \leq j<N-1$, the following implications hold:

(i) If traders $i$ and $j$ disagree with positive probability (i.e., $A_{i j}<1$ ), then traders $i+1$ and $j-1$ disagree for sure (i.e., $A_{i+1, j-1}=0$ ).

(ii) If traders $i+1$ and $j-1$ agree with positive probability (i.e., $A_{i+1, j-1}>0$ ), then traders $i$ and $j$ agree with probability 1 (i.e., $A_{i j}=1$ ).

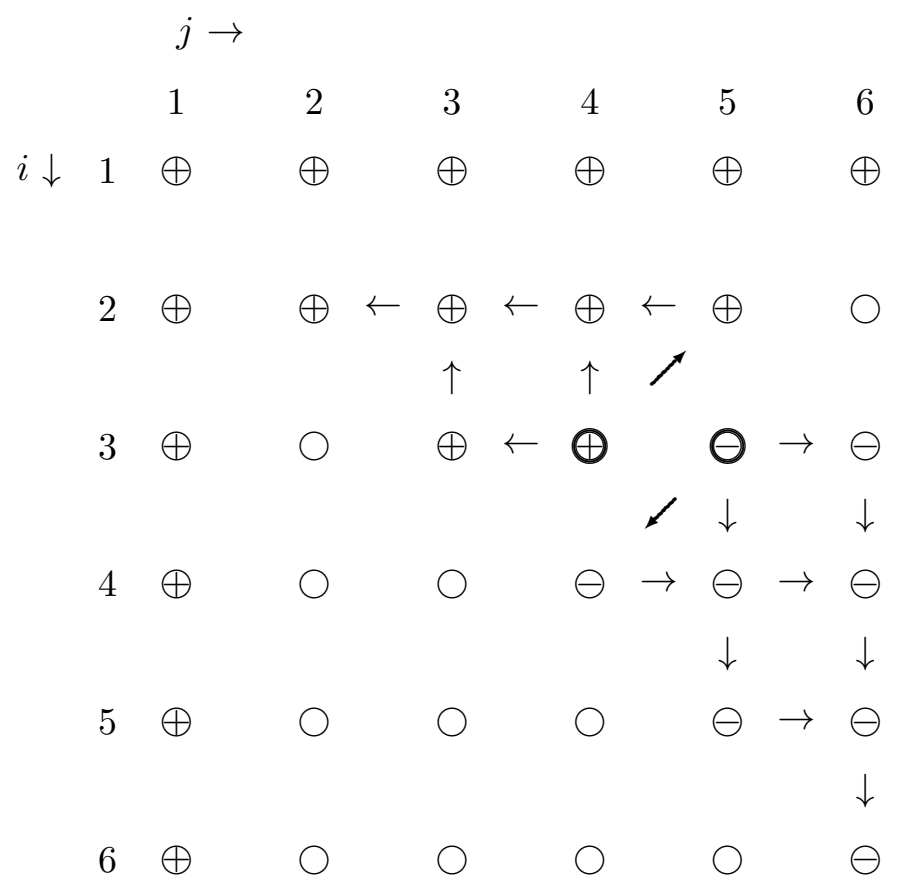

Figure 1: Illustration of Corollary 4.

Agreements are immediate (indicated in Fig. 1 by a "+") when at least one of the partners has an immediate deadline (that is, in the first row and column), or when both have deadline 2, but delay (indicated by "-") may occur when both traders have a more distant deadline. Moreover, when traders $(3,4)$ agree (and thus $\delta\left(v_{2}+v_{3}\right) \leq 1$ ), then traders $(2,5)$ must agree as well, because concavity implies that $\delta\left(v_{1}+v_{4}\right)<\delta\left(v_{2}+v_{3}\right) \leq 1$. Finally, when traders $(3,5)$ disagree (and thus $\delta\left(v_{2}+v_{4}\right) \geq 1$ ), then traders $(4,4)$ must disagree as well, because concavity implies that $\delta\left(v_{3}+v_{3}\right)>\delta\left(v_{2}+v_{4}\right) \geq 1$. This means for the example at hand in Fig. 1 , that 
if one would know that (or if one looks for an equilibrium in which) the pair $(3,4)$ will agree while the pair $(3,5)$ will disagree, then for all other pairs, except the pair $(2,6)$, it is already determined whether their match will end in agreement or disagreement. We use this Corollary for constructing examples in Section 3.4.

\section{3 (Non-)existence of delay}

In this subsection we investigate the necessary and sufficient conditions for the existence of an equilibrium without delay, that is, a stationary subgame perfect equilibrium configuration $(z, w, A)$ where $A_{i j}=1$ for all pairs $(i, j)$. We also characterize the equilibrium strategies precisely for such an equilibrium configuration, and we perform comparative statics exercises.

It is immediate from Definition 1 that no delay implies that $z=p=q$. That is, the stationary distribution of deadlines coincides with the inflow distribution. This is rather intuitive because the no delay assumption implies that no trader remains in the market for more than one period in such an equilibrium.

Suppose there exists a stationary subgame perfect equilibrium configuration without delay. To emphasize the distinct case of no delay, we let $v_{i}(p, \delta)\left(=w_{i}\right)$ denote the expected payoff a trader with deadline $i$ obtains in this equilibrium. When no confusion results we will omit $\operatorname{arguments} p$ and $\delta$. (Again, for convenience we denote $v_{0}=0$.) In an equilibrium without delay, the final condition of Definition 1 reads

$$
v_{i}=\frac{1}{2} \delta v_{i-1}+\frac{1}{2}\left(\sum_{j=1}^{N} p_{j}\left(1-\delta v_{j-1}\right)\right) .
$$

Note that the term between brackets on the right-hand side, which reflects the payoff conditional on being a proposer, does not depend on $i$, the deadline of the proposer. In particular, because $v_{0}=0$, the payoff conditional on being a proposer equals $2 v_{1}$. It follows immediately that for $i>1$

$$
v_{i}=\frac{1}{2} \delta v_{i-1}+\frac{1}{2}\left(2 v_{1}\right)
$$

because with probability $1 / 2$ trader $i$ is offered $\delta v_{i-1}$ and with probability $1 / 2$ he obtains the same payoff that a trader with deadline 1 gets, conditional on being the proposer. It follows that

$$
v_{i}=v_{1}\left(1+\frac{1}{2} \delta+\ldots+\left(\frac{1}{2} \delta\right)^{i-1}\right)=v_{1}\left(1-\left(\frac{1}{2} \delta\right)^{i}\right) /\left(1-\frac{1}{2} \delta\right)
$$

Note that $v_{1}>0$ because otherwise we must have $v_{i}=0$ for all $i$, which is impossible. It follows thus from (2) that $v_{i+1}>v_{i}$ for all $i$. Also note that $v_{1} / v_{N}>1 / 2$. Let $v$ denote 
the expected payoff before knowing one's type. Let $v_{i j}$ denote the expected payoff of trader $i$ conditional on being matched with a trader with deadline $j$ (before knowing whether one is proposer or responder). Because all matches end in agreement, $v_{i j}+v_{j i}=1$. We also have $v=\sum_{i, j} p_{i} p_{j} v_{i j}=\sum_{j, i} p_{j} p_{i} v_{j i}$, so that $2 v=\sum_{i, j} p_{i} p_{j}\left(v_{i j}+v_{j i}\right)=1$, and $v=1 / 2$. Hence,

$$
\frac{1}{2}=\sum_{i=1}^{N} p_{i} v_{i}=v_{1} \sum_{i=1}^{N} p_{i}\left(1+\frac{1}{2} \delta+\ldots+\left(\frac{1}{2} \delta\right)^{i-1}\right) .
$$

Hence,

$$
v_{1}=\frac{1 / 2}{\sum_{i=1}^{N} p_{i}\left(1+\frac{1}{2} \delta+\ldots+\left(\frac{1}{2} \delta\right)^{i-1}\right)}=\frac{1-\frac{1}{2} \delta}{2 W A(p, \delta)}
$$

where

$$
W A(p, \delta)=\sum_{i=1}^{N} p_{i}\left(1-\left(\frac{1}{2} \delta\right)^{i}\right)
$$

denotes a weighted average of powers of $(\delta / 2) . W A(\cdot, \delta)$ defines a dominance order on probability distributions, similar to well-known concepts as first- and second order dominance. In fact, if $p^{\prime}$ first- or second order dominates $p$, then $W A\left(p^{\prime}, \delta\right)>W A(p, \delta) .{ }^{13}$ Note that the expression for the payoff of the type with deadline 1 in an equilibrium without delay only depends on the inflow distribution $p$ insofar as it affects this weighted average $W A(p, \delta)$. We use this result to establish lower and upper bounds on the payoffs that can be obtained in equilibria without delay.

Proposition 5 In an equilibrium without delay, the expected equilibrium payoff of a trader with deadline $j$ equals

$$
v_{j}(p, \delta)=\frac{1-\left(\frac{1}{2} \delta\right)^{j}}{2 \sum_{i=1}^{N} p_{i}\left(1-\left(\frac{1}{2} \delta\right)^{i}\right)} .
$$

Moreover, in an equilibrium without delay $v_{1}>1 / 4, v_{N}<3 / 4$ and $v_{N}<2 v_{1}$.

We know from Corollary 4 that a necessary and sufficient condition for the existence of an equilibrium without delay is that two traders with deadline $N$ have no incentive to disagree, that is, $2 \delta v_{N-1} \leq 1$. It is thus clear that $\delta \leq 2 / 3$ is a sufficient condition for the existence of an equilibrium without delay, because then $2 \delta v_{N-1}<2 \delta v_{N} \leq 2(2 / 3)(3 / 4)=1$.

More accurately, a necessary and sufficient condition for the existence of an equilibrium configuration without delay is given by the following Proposition.

\footnotetext{
${ }^{13}$ This follows from the fact that $h(i)=1-(\delta / 2)^{i}$ is an increasing and concave function of $i$, together with a well-known property of second-order stochastic dominance.
} 
Proposition 6 There exists an equilibrium without delay if and only if

$$
\delta\left(1-\left(\frac{1}{2} \delta\right)^{N-1}\right) \leq W A(p, \delta)
$$

In this case there is exactly one equilibrium without delay. In this equilibrium, a responder of type $j$ accepts any offer $x \geq \delta v_{j-1}$ (and rejects any other offer) whereas a proposer of type $i$ proposes exactly $\delta v_{j-1}$ to a trader of type $j$, where $v_{j}$ is as defined in Proposition 5.

It follows immediately from our equilibrium existence result in Proposition 2 and the previous Proposition that an equilibrium with delay must exist whenever no equilibrium without delay exists. This leaves open the hypothetical possibility that for some parameters both an equilibrium without and an equilibrium with delay exists. We have not found any example where this occurs, and we strongly conjecture that this can never happen. ${ }^{14}$

Corollary 7 In case the inequality in (7) is not satisfied, there exists an equilibrium with delay.

We have observed that $\delta \leq 2 / 3$ is a sufficient condition for an equilibrium configuration without delay to exist. Hence, for delay to exist in the unique equilibrium, the discount factor $\delta$ should not be too low. This is at first sight surprising as in standard models of decentralized trade, disagreement decreases as the delay cost decreases (e.g. Mortensen and Wright, 2002), whereas here the opposite occurs. What happens in the standard models is that there exists both a waiting cost (represented by a low discount factor) and a small participation cost of being active. If all trades were to take place at the competitive price, traders with supramarginal values ${ }^{15}$ would not even participate and all matches would end in agreement. However, if waiting is costly, the participating traders would be willing to buy (sell) at a slightly higher (lower) price. This in turn attracts the supramarginal traders to the market. But when two supramarginal traders meet, no agreement is possible and thus delay occurs when the waiting cost is relatively high. In the current model, all traders can make strictly positive profits from trade and no one would be driven out of the market by small transaction costs.

An interesting feature of our model is that heterogeneous deadlines can arise endogenously in the stationary state even if the inflow distribution does not have much heterogeneity. For example, if the inflow distribution only contains traders with deadlines 1 and $N>2$ and is such

\footnotetext{
${ }^{14}$ For example, it is impossible to have an equilibrium without delay $(z, w, A)$ where $z=p$ and $A_{i j}=1$ for all $i, j$, and, for the same parameters $\delta$ and $p$, an equilibrium in which traders with deadline $N$ disagree with positive probability less than 1 . The latter would yield a stationary distribution $q$ with $W A(q, \delta)<W A(p, \delta)$. Moreover, it is easily established that for an inflow distribution $q$, there would exist an equilibrium without delay with exactly the same payoffs as in the equilibrium with delay. (Such payoffs would satisfy the third condition of the Equilibrium definition.) However, because $W A(q, \delta)<W A(p, \delta)$, the latter equilibrium without delay would yield any type of trader a strictly higher payoff than the original equilibrium without delay, and this is impossible.

${ }^{15}$ That is, buyers with a value below and sellers with a cost above the competitive price.
} 
that delay must occur, then traders with deadline $N-1$ (and possibly lower ones) will arise in equilibrium. Moreover, if there is some friction in the matching process so that not all traders are matched in each period, heterogeneous deadlines will appear in equilibrium even if all new traders have the same deadline $N$. This must necessarily occur if there are more buyers than sellers, for example. Some buyers may not find a match during $N-j \leq N$ periods and will then have a deadline of $j$. On the other hand, it is not possible for delay to occur when all traders are homogeneous and there are no frictions in the matching process. If all traders have deadline $N$ and all are matched, the only reason for disagreement in a match between two deadline $N$ types, is that they expect to get at least one half each in terms of discounted expected payoffs. But if $\delta v_{N-1} \geq 1 / 2$, then $v_{N}>1 / 2$. This would mean that all future generations of traders expect to get strictly more than half of the potential surplus. This is not sustainable and can thus not occur in a stationary subgame perfect equilibrium configuration.

\subsection{Comparative statics and numerical examples}

From the necessary conditions for the existence of an equilibrium without delay in Proposition 6 we obtain immediately

Corollary 8 Suppose there exists a stationary subgame perfect equilibrium configuration without delay when the inflow distribution is $p=\left(p_{1}, \ldots, p_{N}\right)$ (where $p_{N}>0$ ) and the discount factor equals $\delta$.

(i) Let $p^{\prime}$ first-order stochastically dominates $p$. When the distribution shifts from $p$ to $p^{\prime}$, a stationary subgame perfect equilibrium configuration without delay still exists and $v_{j}\left(p^{\prime}, \delta\right)<$ $v_{j}(p, \delta)$ for all $j$.

(ii) Let $p^{\prime}$ second-order stochastically dominates $p$. When the distribution shifts from $p$ to $p^{\prime}$, a stationary subgame perfect equilibrium configuration without delay still exists and $v_{j}\left(p^{\prime}, \delta\right)<v_{j}(p, \delta)$ for all $j$.

(iii) When the discount factor decreases from $\delta$ to $\delta^{\prime}$, there still exists a stationary subgame perfect equilibrium configuration without delay. Moreover, the difference in payoff $\Delta_{j}=$ $v_{j}\left(p, \delta^{\prime}\right)-v_{j}(p, \delta)$ is decreasing in $j$. In particular, $\Delta_{1}>0$ and $\Delta_{N}<0$.

(iv) Suppose that all traders in the inflow distribution have either deadline $N$ or $N-1$. Then there exists a unique stationary subgame perfect equilibrium configuration, and there is no delay in it. 
These results are quite intuitive and straightforward. Results (i) and (ii) follow from the observation that $W A\left(p^{\prime}, \delta\right) \geq W A(p, \delta)$ when $p^{\prime}$ first- or second-order stochastically dominates $p$. Delay is more likely to occur when deadlines are more heterogenous, that is when the inflow distribution is more risky. The fact that in equilibria without delay average payoff remains the same (at one half) whereas all types of traders suffer from a shift from $p$ to $p^{\prime}$ seems odd at first but is just an instance of Simpson's paradox: all payoffs are reduced but when more weight is placed on the (higher) ones for high deadline traders, the average does not change. Result (iii) follows from the observation that $W A(p, \delta)$ is decreasing in $\delta$. Delay is less likely to occur when it is more costly. When the discount factor is close to zero, all traders get similar payoffs. An increase of the discount factor strengthens the bargaining position of traders with long deadlines and weakens it for those with short ones, and thus increases the payoff inequality between different traders.

The corollary indicates that equilibrium configurations with a positive amount of delay can exist only if both (i) a sufficiently large proportion of traders has a short deadline and (ii) there are traders that are sufficiently patient. Traders with a high deadline are then likely to be matched with traders with short deadlines, and are thus willing to wait when by bad luck they are matched with other traders with high deadlines. On the other hand, when many traders have short deadlines, the probability of delay will be small. Also, when traders have a high discount factor, the cost of delay is rather small. In order to get some more insight in the probability and cost of delay caused by heterogeneous deadlines, we will consider a special case with inflow distribution putting positive weight only on deadlines 1 and 3. We will first illustrate how the amount of delay and payoffs in the equilibrium configuration varies with the discount factor. Then we will consider how these measures vary with the inflow distribution. In these examples equilibrium configurations are unique: we have looked for all equilibria with any of the possible delay configurations consistent with Corollary 4, and always found exactly one.

Example 1. Suppose $N=3$ and the inflow distribution is $p=(0.75,0,0.25)$. For any discount factor $\delta \in[0,1]$ there exists a unique stationary subgame perfect equilibrium configuration. The amount of delay increases in $\delta$. There exist thresholds $\delta_{1} \approx 0.813, \delta_{2} \approx 0.822$, $\delta_{3} \approx 0.963$, and $\delta_{4} \approx 0.964$ such that the stationary subgame perfect equilibrium configuration is characterized by

- For $\delta<\delta_{1}$, there is no delay at all.

- For $\delta \in\left(\delta_{1}, \delta_{2}\right)$, there is random delay only between two traders who have deadline 3 .

- For $\delta \in\left(\delta_{2}, \delta_{3}\right)$, there is deterministic delay between two traders who have deadline 3 . 


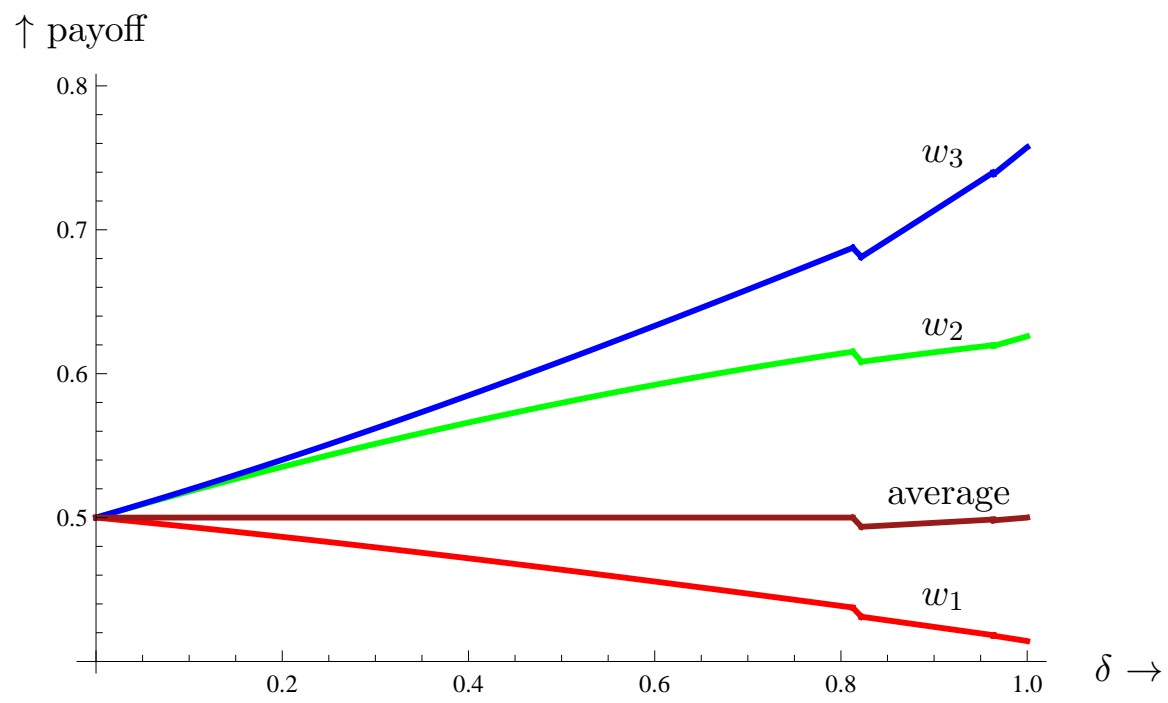

Figure 2: Equilibrium payoffs for the three types, and average payoff for new trader.

- For $\delta \in\left(\delta_{3}, \delta_{4}\right)$, there is deterministic delay between two traders who have deadline 3 and random delay between traders with deadlines 2 and 3 .

- For $\delta>\delta_{4}$, there is deterministic delay between traders with deadlines 2 and 3 , and between traders with deadlines 3 and 3 .

Fig. 2 plots the payoffs of the three different types in the stationary subgame perfect equilibrium configuration as a function of the discount factor. At $\delta=0$ the payoffs coincide because this effectively means that every trader must make an agreement immediately. As the discount factor increases, the payoffs diverge in the region where no delay occurs. In the regions where in some pairs the probability of agreement is strictly between 0 and 1, payoffs decrease, even when the discount factor increases. ${ }^{16}$ Average payoff is non-monotonic in $\delta$ : It equals one half when $\delta$ is small (no delay) and when $\delta=1$ (costless delay). In an intermediate region average payoff is below one half because there is costly delay.

Example 2. Let $N=3$ and $\delta=0.98$. For any inflow distribution $p=\left(p_{1}, 0,1-p_{1}\right)$ there exists a unique stationary subgame perfect equilibrium configuration. Even though $W A(p, \delta)$ is monotonically decreasing in $p_{1}$, neither the amount of delay nor the average payoff of new traders flowing into the market is monotonic in $p_{1}$. There exist thresholds $\rho_{1} \approx 0.37, \rho_{2} \approx 0.382$, $\rho_{3} \approx 0.7247$, and $\rho_{4} \approx 0.7252$ such that the stationary subgame perfect equilibrium configuration is characterized by

- For $p_{1}<\rho_{1}$, there is no delay at all.

\footnotetext{
${ }^{16}$ This happens for $\delta \in\left(\delta_{1}, \delta_{2}\right)$ and $\delta \in\left(\delta_{3}, \delta_{4}\right)$. The latter is difficult to notice in the graph because the interval is very short.
} 


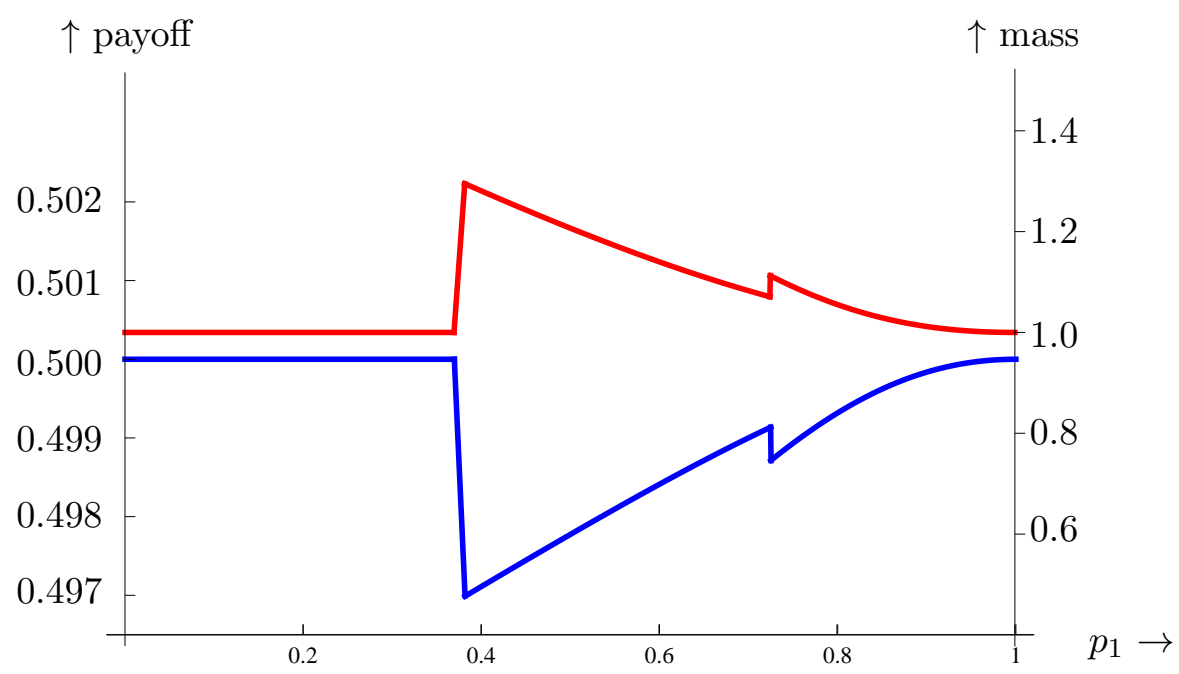

Figure 3: Total mass of buyers (or sellers) (top) and average equilibrium payoff of a new trader (bottom) when inflow distribution equals $p=\left(p_{1}, 0,1-p_{1}\right)$ and $\delta=0.98$.

- For $p_{1} \in\left(\rho_{1}, \rho_{2}\right)$, there is random delay only between two traders who have deadline 3 .

- For $p_{1} \in\left(\rho_{2}, \rho_{3}\right)$, there is deterministic delay between two traders who have deadline 3 .

- For $p_{1} \in\left(\rho_{3}, \rho_{4}\right)$, there is deterministic delay between two traders who have deadline 3 and random delay between traders with deadlines 2 and 3 .

- For $p_{1}>\rho_{4}$, there is deterministic delay between traders with deadlines 2 and 3 , and between traders with deadlines 3 and 3 .

Fig. 3 plots the expected equilibrium payoff of a new trader flowing into the market, and the total mass of traders in equilibrium. In the regions where a pure equilibrium exists (that is, where agreements in a pair occur with probability 0 or 1 ), the average payoff is increasing in $p_{1}$. Clearly, a higher probability of being matched with a deadline 1 trader improves the average payoff. However, in the region where some agreements occur with probability strictly between 0 and 1 average payoffs are decreasing. In this region the mass of traders with deadline 2 increases sharply, which reduces the proportion of deadline 1 traders, which has a negative effect on average payoffs. Clearly, the highest average payoff of 0.5 is obtained when there is no delay $\left(p_{1}<\rho_{1}\right)$ or when all traders have deadline $1\left(p_{1}=1\right)$. The largest mass of traders occurs at $p_{1}=\rho_{2}$ where there is deterministic disagreement only between traders with deadline 3 .

\subsection{Asymmetric markets}

We have assumed throughout the section that the number of sellers and buyers flowing into the market is the same. We also assumed that the distribution of deadlines is the same for buyers 
and sellers. This symmetry assumption allowed us to simplify the exposition. However, in order to study the effects of a change in the mass or the type distribution on one market side, we need to study asymmetric markets. In this subsection we discuss briefly how this can be done. We restrict attention to equilibrium configurations without disagreements and show how payoffs are affected by such changes on one side of the market.

Let $p_{i}^{S}$ denote the mass of sellers with deadline $i$ flowing into the market, and let $(1+b) p_{j}^{B}$ denote the mass of buyers with deadline $j$ flowing into the market. Without loss of generality we may assume that $b \geq 0, \sum_{i=1}^{N_{S}} p_{i}^{S}=1$ and $\sum_{j=1}^{N_{B}} p_{j}^{B}=1$. If $b>0$ then the buyers form the long side of the market. We will assume that then each seller will be matched with one buyer. Obviously, in this case there will always be buyers who will not get matched in any single round. That means that there will certainly be buyers who will not immediately close a deal. In fact some buyers will miss their deadline, because they may never be matched with a seller before their deadline expires. However, we will be interested in the possibility of delay on the (short) side of the sellers. That is, will there be disagreement in some pair of matched traders?

The definition of a stationary subgame perfect equilibrium configuration can be generalized in a straightforward manner. Namely, it will be a tuple $\left(z^{B}, z^{S}, w^{B}, w^{S}, E\right)$, where $z_{j}^{B}$ and $z_{i}^{S}$ denote the mass of buyers (sellers) with deadline $j(i)$ and where $w_{j}^{B}$ and $w_{i}^{S}$ denote the expected payoff of a buyer (seller) with deadline $j(i)$. The $N_{B} \times N_{S}$ matrix $E$ indicates the probability of agreement in a pair where buyer $j$ and seller $i$ are matched. If $\sum_{i=1}^{N_{S}} z_{i}^{S}=1+T$ and $T>0$, there will be delay among sellers. The stationarity condition for buyers must now take into account not only that some buyers remain from the previous period because they made or rejected unacceptable proposals, but also those buyers who were not matched in the last period. If $B$ denotes the mass of buyers in a stationary state, the probability of not being matched equals $1-(1+T) / B$.

The existence of a stationary subgame perfect equilibrium configuration can be shown, but we omit the proof. In this section we will restrict ourselves to analyzing equilibria without disagreements. We will derive the necessary condition for existence of such an equilibrium configuration and we perform comparative statics on the payoffs of traders in such a configuration with respect to $b$ and with respect to the distribution of deadlines.

Let $\left(z^{B}, z^{S}, w^{B}, w^{S}, E\right)$ be a stationary subgame perfect equilibrium configuration in which agreement occurs in all pairs formed. Then obviously $z_{i}^{S}=p_{i}^{S}$ for all $i$ and $\sum_{i=1}^{N_{S}} z_{i}^{S}=1$. The stationary distribution in an equilibrium configuration without disagreements obviously satisfies $q_{i}^{S}=p_{i}^{S}$. 
On the other hand, for buyers we have $z_{N_{B}}^{B}=(1+b) p_{N_{B}}^{B}$ and for $j<N_{B}$ we have

$$
z_{j}^{B}=(1+b) p_{j}^{B}+z_{j+1}^{B}(1-1 / B)
$$

where $B=\sum_{j=1}^{N_{B}} z_{j}^{B}$. It follows that

$$
\frac{z_{j}^{B}}{b+1}=\sum_{k=j}^{N_{B}} p_{k}^{B}(1-1 / B)^{k-j} .
$$

Summing over all $z_{j}^{B}$, one obtains $B=1+b+\left(B-z_{1}^{B}\right)(1-1 / B)$. It is thus easily verified that, for given and fixed $b, B$ can be computed as the unique root of

$$
\sum_{j=1}^{N_{B}} p_{j}^{B}(1-1 / B)^{j}=b /(b+1) .
$$

Note that if $b=0, B=1$ whereas if $b>0, B>1$. (In fact, $B>b+1$ as long as $p_{1}^{B}<1$.) The stationary distribution of deadlines of buyers will now differ from the inflow distribution whenever $b>0$. Namely, $q_{j}^{B}=z_{j}^{B} / B$. In particular, $q_{N_{B}}^{B}<p_{N_{B}}^{B}$ when $b>0$.

Note that the expected payoff of a seller with deadline $i+1$ satisfies $w_{i+1}^{S}=\frac{1}{2} \delta w_{i}^{S}+\frac{1}{2}\left(2 w_{1}^{S}\right)$, as the first term reflects the payoff as a responder, and the second term reflects the payoff as a proposer, which is the same for all sellers. Hence, as before, we have

$$
w_{i}^{S}=w_{1}^{S} \frac{1-(\delta / 2)^{i}}{1-\delta / 2}
$$

Similarly, the expected payoff of a buyer with deadline $j+1$ (before knowing whether he will be matched or not) satisfies $w_{j+1}^{B}=\delta w_{j}^{B}(1-1 /(2 B))+1 /(2 B)(2 B) w_{1}^{B}$. It follows that

$$
w_{j}^{B}=w_{1}^{B} \frac{1-\left[\left(1-\frac{1}{2 B}\right) \delta\right]^{j}}{1-\left(1-\frac{1}{2 B}\right) \delta} .
$$

Of course, we also have

$$
w_{1}^{S}=\frac{1}{2} \sum_{j=1}^{N_{B}} q_{j}^{B}\left(1-\delta w_{j-1}^{B}\right)
$$

and

$$
w_{1}^{B}=\frac{1}{2 B} \sum_{i=1}^{N_{S}} q_{i}^{S}\left(1-\delta w_{i-1}^{S}\right) .
$$

\section{Equal mass, different distributions.}

Let us now consider the special case where the mass of buyers and sellers is the same (i.e. 
$b=0$ and $B=1$ ) but where the distributions of deadlines of buyers and sellers are distinct.

It is straightforward to establish the following result about the payoffs that different traders get, conditional on playing an equilibrium configuration without delay.

Proposition 9 In an equilibrium configuration without delay, the payoffs of different traders is given by

$$
\begin{aligned}
w_{1}^{S} & =\frac{(2-\delta)\left(2-\delta-W A\left(p^{B}, \delta\right)\right)}{\left.2\left((2-\delta)\left(W A\left(p^{B}, \delta\right)+W A\left(p^{S}, \delta\right)\right)-2 W A^{(} p^{B}, \delta\right) W A\left(p^{S}, \delta\right)\right)} \\
w_{1}^{B} & =\frac{(2-\delta)\left(2-\delta-W A\left(p^{S}, \delta\right)\right)}{\left.2\left((2-\delta)\left(W A\left(p^{B}, \delta\right)+W A\left(p^{S}, \delta\right)\right)-2 W A^{(} p^{B}, \delta\right) W A\left(p^{S}, \delta\right)\right)} \\
w_{i}^{S} & =w_{1}^{S} \frac{1-(\delta / 2)^{i}}{1-\delta / 2} \\
w_{i}^{B} & =w_{1}^{B} \frac{1-(\delta / 2)^{i}}{1-\delta / 2} .
\end{aligned}
$$

It follows that

$$
\frac{w_{i}^{S}}{w_{i}^{B}}=\frac{2-\delta-W A\left(p^{B}, \delta\right)}{2-\delta-W A\left(p^{S}, \delta\right)}
$$

Hence, $w_{i}^{S}>w_{i}^{B}$ if and only if $W A\left(p^{S}, \delta\right)>W A\left(p^{B}, \delta\right)$. In particular, sellers make higher profits than buyers when the distribution of deadlines of sellers first- or second-order dominates the distribution of deadlines of buyers. In other words, it is advantageous to belong to the side of the market with higher and more dispersed deadlines.

The existence of an equilibrium configuration without delay requires that $\delta\left(w_{N-1}^{S}+w_{N-1}^{B}\right) \leq$ 1. This condition can be rewritten as

$$
\delta\left(1-(\delta / 2)^{N-1}\right) \leq \frac{(2-\delta)\left(W A\left(p^{B}, \delta\right)+W A\left(p^{S}, \delta\right)\right)-2 W A\left(p^{B}, \delta\right) W A\left(p^{S}, \delta\right)}{4-2 \delta-\left(W A\left(p^{B}, \delta\right)+W A\left(p^{S}, \delta\right)\right)} .
$$

\section{Equal distributions, different mass.}

We now consider the case where the distribution of deadlines is the same for buyers and sellers, but where one side of the market (buyers) has more participants.

It is clear that when the ratio of buyers to sellers converges to infinity, the probability of being matched goes to zero for buyers, so that the expected payoffs for buyers, independently of their deadline, will converge to zero as well. In this case the payoff of a seller with deadline $i$ converges to $\frac{1}{2} \frac{1-(\delta / 2)^{i}}{1-\delta / 2}$. In particular, the most patient seller receives a payoff of at most $1-(1 / 2)^{N}<1$. It is thus also clear that an equilibrium configuration where all matches end in agreement exists and is in fact the unique equilibrium configuration. It follows from the above considerations that for $b$ large enough, all matches end in agreement. 


\section{Equilibrium Analysis under Private Information about Dead- lines}

Thus far we assumed that traders within a match know each other's deadline, so that bargaining is under perfect information. In this section we assume that each trader only knows his own deadline, but not the one of his matched partner. This naturally affects the strategies available to a trader. Given our restriction to stationary subgame perfect equilibrium configurations, a pure strategy of a trader must specify the offer she will make and the offers she will accept when her own deadline is $i$. For a given stationary distribution of deadlines, $q$, and for given traders' strategies, one can compute the expected payoffs for a trader with deadline $i$. We will denote this by $v_{i}$. Note that because a trader with deadline $i+1$ can mimic one with deadline $i$, we must have $v_{i} \leq v_{i+1}$.

In a stationary equilibrium state it must be that a trader with deadline $i$ accepts any proposal that gives her $x>\delta v_{i-1}$ and rejects any proposal that gives her $x<\delta v_{i-1}$, where $v_{0}=0$. When the proposal is exactly equal to $\delta v_{i-1}$, the responder is indifferent and, in principle, may use a mixed strategy. However, when the responder is indifferent and accepts with probability strictly less than one, the proposer (with deadline $j$ ) could deviate and offer slightly more, which would then be accepted for sure. If this is profitable for the proposer, it must be the case, in equilibrium, that the indifferent responder accepts $\delta v_{i-1}$ with probability 1 . On the other hand, if the proposer does not gain from offering slightly more, it must be the case that the proposer is in fact indifferent between the responder accepting or rejecting. This implies that $1-\delta v_{i-1}=\delta v_{j-1}$. (Note that this cannot happen when $i=1$.) But in this case it is profitable for the proposer to make a strictly lower proposal $\delta v_{k-1}<\delta v_{i-1}$. In particular, offering the largest $\delta v_{k-1}<\delta v_{i-1}$ will be a strict improvement. Hence, in an equilibrium configuration, it must be the case that any equilibrium proposal is accepted with probability one by responders that are indifferent.

The proposals that are made in a stationary equilibrium state must be in the set $X=$ $\left\{\delta v_{0}, \ldots, \delta v_{N-1}\right\}$. Namely, offers strictly above $\delta v_{i-1}$ will be accepted for sure by a trader with deadline $i$ or lower, and therefore it is never optimal for a proposer to make such offers. Because it is possible that $v_{i}=v_{j}$ for some $i \neq j$, we will write also $X=\left\{x_{1}, \ldots, x_{n}\right\}$ with the understanding that $x_{k}<x_{k+1}$. It turns out that we will have to allow for proposers randomizing between different proposals. Let $\Delta(X)$ denote the set of probability distributions on a finite set $X$.

We are now ready to formally define a stationary subgame perfect equilibrium configuration for the case where deadlines are private information. 
Definition 10 We call $(z, v, s)=\left(\left(z_{1}, \ldots ., z_{N}\right),\left(v_{1}, \ldots, v_{N}\right), s\right) \in \Re_{+}^{N} \times \Re_{+}^{N} \times \Delta(X)^{N}$ a stationary subgame perfect equilibrium configuration if the following holds:

Stationarity $z_{N}=p_{N}$ and for all $i<N$

$$
z_{i}=p_{i}+\frac{1}{2} z_{i+1}\left[\sum_{k}\left[s_{i+1}\left(x_{k}\right) \sum_{j: \delta v_{j-1}>x_{k}} q_{j}\right]\right]+\frac{1}{2} z_{i+1}\left[\sum_{j}\left[q_{j} \sum_{k: \delta v_{i}>x_{k}} s_{j}\left(x_{k}\right)\right]\right]
$$

where $q_{i}=z_{i} /(1+T)$.

\section{Expected Payoffs For all $i$}

$$
\begin{aligned}
v_{i}= & \frac{1}{2} \sum_{k}\left[s_{i}\left(x_{k}\right)\left(\left(1-x_{k}\right) \sum_{j: \delta v_{j-1} \leq x_{k}} q_{j}+\delta v_{i-1} \sum_{j: \delta v_{j-1}>x_{k}} q_{j}\right)\right] \\
& +\frac{1}{2} \sum_{j} q_{j}\left[\sum_{k} \max \left\{\delta v_{i-1}, x_{k}\right\} s_{j}\left(x_{k}\right)\right] .
\end{aligned}
$$

\section{Proposal Probabilities}

$$
s_{i}\left(x_{k}\right)>0 \text { implies } x_{k} \in \arg \max _{x_{l}}\left\{\left(1-x_{l}\right) \sum_{j: \delta v_{j-1} \leq x_{l}} q_{j}+\delta v_{i-1} \sum_{j: \delta v_{j-1}>x_{l}} q_{j}\right\} .
$$

The stationarity condition and the expected payoff condition are similar to the case of bargaining under perfect information. The acceptance probabilities condition under perfect information is replaced here by one of proposal probabilities. It states that only offers that maximize a proposer's expected payoff can be chosen with positive probability. We need the possibility of random proposals in order to show the existence of an equilibrium configuration, employing the Kakutani fixed point theorem.

Proposition 11 For any inflow distribution $p$ and any discount factor $\delta \in(0,1]$ there exists a stationary subgame perfect equilibrium configuration.

Characterizing no-delay equilibrium configurations is straightforward in the case of bargaining under imperfect information.

Proposition 12 For $N>1$ there exists a stationary subgame perfect equilibrium configuration with $v_{i}=v$ for all $i$ if and only if $p_{1} \leq 2(1-\delta) /(2-\delta)$. In this case $v=1 / 2$ and no delay occurs.

The intuition for this result is as follows. If all traders obtain the same payoff in equilibrium, this payoff must equal one half and all proposers offer $\delta / 2$. Given those proposer strategies, only a responder with deadline 1 would accept (any) offer below $\delta / 2$. But when the probability of 
being matched with a trader with deadline 1 is sufficiently small, it is not optimal to make a very greedy offer of zero.

Proposition 13 Suppose $1>p_{1}>2(1-\delta) /(2-\delta)$. In any stationary subgame perfect equilibrium configuration there will be delay. The payoffs are strictly increasing in deadlines. There exist inflow distributions for which deadlines will be missed in equilibrium.

The intuition for this result is as follows. When the probability of being matched with a trader with deadline 1 is sufficiently high, it is optimal for the trader with the highest deadline to make the greedy zero offer, which will only be accepted by traders whose deadline is about to expire. Thus, in this case delay occurs whenever a trader with the highest deadline is chosen as the proposer and is being matched with a trader with a deadline that is not about to expire. This in turn implies that a trader with deadline $i+1$ will obtain a strictly higher expected payoff than a trader with deadline $i$, as the first can mimic the latter for $i$ periods, but refuse the greedy offer in the $i$-th period. He will then have one extra opportunity to obtain a strictly positive payoff. Of course, the condition in the Proposition is equivalent to $\delta>2\left(1-p_{1}\right) /\left(2-p_{1}\right)$. Hence, for any distribution with full support there will be delay if traders have a discount factor close to 1 .

Deadlines are missed only when the proposer has deadline 1 and finds it optimal to make a proposal that the traders with the longest deadlines will reject. This will only occur when there are not too many traders with the longest deadline. Clearly, the fraction of traders in the market that will miss their deadline in the next period is thus bounded above by 12.5 per cent. Namely, the probability that a random trader in the market will miss his deadline in the next instant is bounded above by $q_{1}\left(1-q_{1}\right) / 2 \leq 0.125$. However, the probability that a trader will eventually miss his deadline may even be higher than that, because traders with high deadlines may first delay trade and later miss their deadline.

Next we show that in any stationary subgame perfect equilibrium configuration the proposals made are weakly decreasing in the deadline of the proposer. That is, the more patient a trader is, the greedier his offers will be. Or phrased alternatively, the offers made by one particular trader become more generous over time.

Proposition 14 In any stationary subgame perfect equilibrium configuration (with delay) the equilibrium offers are weakly monotonically decreasing in deadline: If type $j$ weakly prefers offering $\delta v_{i-k}$ rather than $\delta v_{i}$ (for $k>0$ ), then type $j+1$ strictly prefers offering $\delta v_{i-k}$ rather than $\delta v_{i}$. 
We can use the preceding results to characterize the equilibria in more detail. There are three possible types of stationary subgame perfect equilibrium configurations: (1) configurations without delay, (2) configurations with delay but without deadlines being missed, and (3) configurations with delay and deadlines being missed. The first type of subgame perfect equilibrium configurations has all traders make the same offer of $\delta / 2$ which is immediately accepted. Clearly, all traders make the same expected profit and there is no advantage of being more patient. Such a configuration exists only if the fraction of traders with the shortest deadline is (very) small, so that no trader has an incentive to ask for the whole pie.

The second and third type of configurations do exhibit an advantage of having a longer deadline as expected payoffs are strictly increasing in deadline. When no deadlines are missed, a proposer with deadline 1 will make an offer that all responders will accept, that is, he will offer $\delta v_{N-1}$. Hence, when a trader with deadline $N$ is matched with a deadline 1 trader, there will be immediate agreement. However, if a trader with deadline $N$ is matched with any other type of trader, there is a probability of at least one half that no agreement will result. Namely, if a trader with deadline 2 makes an acceptable proposal to $N$, then it is implied that $N$ will always make a greedy offer of zero, which a trader with deadline 2 (or higher) will certainly reject. This is so because otherwise traders with deadlines 1 and 2 would achieve the same payoff, which is impossible from Proposition 13. In the third type of equilibrium configuration, a trader with deadline $N$ will even reject the proposal by a trader with deadline 1 . Hence, whenever the trader with deadline $N$ is the responder (which occurs with probability one half), no agreement results. These observations indicate that the amount of delay and unsuccessful bargaining is particularly high when the inflow distribution consists mainly of traders with the highest deadline and a few with the lowest deadline (but just enough to make it worthwhile for the patient traders to make extremely greedy offers).

\section{$5 \quad$ Related literature}

Even though our paper is unique in considering non-stationary preferences, it is related to two strands of literature. First, there is the literature that addresses the effects of a common deadline in bilateral bargaining. Second, there is a large literature on decentralized trade by means of matching and bargaining. We discuss these strands of literature, and their relation with this paper, in turn. 


\subsection{Deadlines in bargaining}

This literature considers two person bargaining with a common deadline. This starts with the finite horizon version of the Rubinstein bargaining model (Ståhl, 1972), which shows that the first mover advantage decreases with the number of bargaining rounds. Yildiz (2004) explains why agreements are often reached close to the deadline when the deadline is fixed, but that agreement is immediate in case of stochastic deadlines. Ma and Manove (1993) consider a model of bargaining in which players can time their proposals, but in which the offers are received with some random delay. They show that players start by delaying making proposals, and then make proposals that are sometimes rejected. Agreements tend to be reached near the deadline and sometimes no agreement is reached before the deadline expires. Ponsati (1995) analyzes a bargaining game between two players over two possible outcomes. The players have opposed preferences about the outcomes but the exact utility the players experience from the outcomes is private information. Ponsati (1995) shows that many concessions are made exactly at the deadline but not just before (but possibly much earlier). The deadline may also be missed altogether. Fershtman and Seidmann (1993) assume that bargainers are committed not to accept a proposal that is worse than a previously rejected proposal and show that agreements will only be reached at the deadline.

\subsection{Dynamic matching and bargaining}

Our paper also relates to the large literature on dynamic matching and bargaining, starting with Rubinstein and Wolinsky (1985) and summarized in Gale (2000). A dynamic matching and bargaining game constitutes a natural model of decentralized trade when there are many traders on both sides of the market. Many papers in this literature analyze whether the competitive equilibrium outcome is obtained when frictions become negligible (e.g., Gale (1987) and Satterthwaite and Shneyerov, 2007). A paper closely related to ours is Bose (1996). He considers a dynamic matching and bargaining market in which traders have either a low or a high discount factor. The basic idea that patient traders, when matched together, may prefer to wait until matched with impatient traders, is similar. However, Bose's (1996) model does not allow for a long and a short side of the market. Whereas in our model preferences and behavior of a particular trader are non-stationary, in Bose's model they are stationary. Anwar and Sákovics (2007) consider a model in which the good is perishable, which puts pressure on the seller to agree to lower prices. They assume that with some given probability the good goes bad at the end of each period and the seller disappears from the market. In case this probability equals one, it is as if sellers have a deadline of 1 and buyers have no deadline. They show 
that inefficiencies arise because of excess demand (i.e., buyers have to queue to be matched) in the limiting steady state. In our (symmetric) model all traders are matched and could reach immediate agreement, but pairs of patient traders actually prefer and agree to delay and wait for more profitable opportunities.

The role of heterogeneous time preferences played in Bose (1996) and our paper is similar to the one of heterogeneous valuations. Samuelson (1992) considers buyers and sellers with heterogeneous valuations and shows that two traders who could realize a positive surplus from trading, may decide to break up negotiations and look for alternative partners, with which they can make even more profitable agreements. The current paper has in common with Samuelson (1992) that the disagreement point of a pair of traders is endogenously determined by the outside options generated by the market, and that this is different from the one of the bargaining problem studied in isolation. A difference is that in Samuelson (1992) the surplus to be divided depends on the actual match and is not known, whereas the disagreement payoff for a particular trader is constant over time. ${ }^{17}$ Jackson and Palfrey (1998) consider a market where traders with heterogeneous valuations can return after disagreement, but without new traders flowing in. They show that for a robust set of distributions of buyer and seller valuations the constrained efficient trading rule is not attainable. The intuition for this is that trades in one period create an externality on the distribution of traders who are rematched in the next period. In a similar framework, Moreno and Wooders (2002) show that although trading patterns may be inefficient, the welfare loss due to inefficient trading vanishes when frictions are small. Similar to Jackson and Palfrey (1998), Damiano et al. (2005) consider a dynamic matching model where the matching value function displays complementarities and where a fixed set of firms and workers have a finite number of periods in order to form a match. They show that a small participation cost in each round eliminates the sorting function of the market because almost all matches are formed in the first round.

\section{Conclusions}

We have shown that, in the context of a dynamic matching and bargaining market, heterogeneous deadlines can be successfully incorporated to model situations where traders have different degrees of time pressure. Our model captures that many real-life bargaining situations are framed in terms of deadlines ("When do I need to have an agreement?") rather than in terms

\footnotetext{
${ }^{17}$ Mortensen and Wright (2002), Satterthwaite and Shneyerov (2007), and Shneyerov and Wong (2010b) also allow for distributions of buyer's valuations and seller's costs in a dynamic matching and bargaining model, but their main focus is on the convergence of equilibria of such model to Walrasian equilibria as frictions (such as transaction costs) disappear.
} 
of discounting ("How much am I willing to sacrifice to have an agreement today rather than tomorrow?"). Stationary equilibria are shown to exist, for any initial distribution of trader's deadlines.

When proposers have perfect information about the responder's deadline, the steady state equilibrium has the intuitive property that the more patient traders receive higher expected payoffs. This may induce these traders to delay agreement when matched with similar traders. In an equilibrium with delay a trader with a long deadline may initially reject proposals, but later on he will accept the same or worse offers, because his outside option deteriorates over time. This a realistic feature of bargaining, for example in the real estate market (see, e.g., Merlo and Ortalo-Magné, 2004). Compared to the initial inflow distribution of deadlines, in an equilibrium with delay the steady state distribution is shifted towards longer deadlines. This negatively affects all traders, but in particular those who are very much pressed by time. A directed mechanism that matches traders with the same deadline would remove any delay and, moreover, would guarantee all traders the same payoff. The availability of such a mechanism would attract, in first instance, the exploited traders with short deadlines. An unravelling argument then shows that in fact all trade will take place in this directed matching market. We refer the reader to our previous working paper version (Hurkens and Vulkan, 2006) for details.

Delay may occur exactly because traders are willing to wait until matched with a trader with a short deadline. One may thus have conjectured that delay cannot occur when deadlines are private information. However, when proposers do not observe the deadline of the responder, equilibria without delay only exist when the probability of having deadline 1 is small. In this case all traders obtain the same payoff. When the probability of having deadline 1 is relatively high, many proposers are willing to take a chance and make very greedy offers and the equilibrium does exhibit delay. In fact, deadlines are missed when even proposers with deadline 1 make such greedy offers (but are matched with more patient responders).

It should be mentioned that although our model deals with buyers and sellers, it can also be applied to other settings of two-sided matching markets, such as workers and firms, authors and co-authors, or men and women. ${ }^{18}$ In these situations one usually assumes that the surplus is not fixed but depends on the characteristics of the partners. The main subject of study is then to determine who matches up with whom. For example, will there be positive assortive matching whenever this is efficient? Clearly, our model can and needs to be enriched in order to deal with these type of questions. It seems plausible that the introduction of heterogeneous deadlines will affect these matching models in similar ways. Delay may occur and inefficient

\footnotetext{
${ }^{18}$ See, e.g., Shimer and Smith (2000) and Damiano et al. (2005).
} 
matches may form whenever the partners are pressed by time. However, a full analysis of this situation is beyond the scope of the present paper and is left for future research.

\section{Appendix}

\section{Proof of Proposition 2}

Let $Z=\left\{z \in \Re_{+}^{N}: \sum_{i} z_{i} \geq 1\right.$ and $\left.z_{i} \leq N+1-i\right\}$ and let $W=\left\{w \in \Re_{+}^{N}: w_{1} \leq w_{2} \leq \ldots \leq\right.$ $\left.w_{N} \leq 1\right\}$. Let $\mathcal{M}$ denote the set of all symmetric $N \times N$ matrices with entries in the interval $[0,1]$. Consider the following correspondence $G: Z \times W \rightarrow Z \times W \times \mathcal{M}$ :

$$
\begin{aligned}
G(z, w)= & \left\{(z, w, A): A_{i j}=0 \text { if } \delta\left(w_{i-1}+w_{j-1}\right)>1\right. \text { and } \\
& \left.A_{i j}=1 \text { if } \delta\left(w_{i-1}+w_{j-1}\right)<1\right\}
\end{aligned}
$$

and the following mapping $H: Z \times W \times \mathcal{M} \rightarrow Z \times W$ :

$$
H(z, w, A)=(\tilde{z}, \tilde{w})
$$

where

$$
\tilde{z}_{N}=p_{N}, \tilde{z}_{i}=p_{i}+\frac{z_{i+1}}{\sum_{k} z_{k}}\left(\sum_{j=1}^{N} z_{j}\left(1-A_{i+1 j}\right)\right) \text { for } i<N
$$

and

$$
\tilde{w}_{i}=\frac{1}{2} \delta w_{i-1}+\frac{1}{2 \sum_{k} z_{k}}\left(\sum_{j=1}^{N} z_{j}\left(\max \left\{\delta w_{i-1}, 1-\delta w_{j-1}\right\}\right) .\right.
$$

Note that $\tilde{z}_{i} \leq p_{i}+z_{i+1} \leq 1+N+1-(i+1)=N+1-i$ and that $\tilde{w}_{i} \leq \tilde{w}_{i+1} \leq 1$ when $w_{i-1} \leq w_{i}$ so that $H$ really maps into $Z \times W$.

We now combine $G$ and $H$ to construct a correspondence $F: Z \times W \rightarrow Z \times W$ as follows:

$$
F(z, w)=\{H(z, w, A):(z, w, A) \in G(z, w)\}
$$

$F$ is an upper semi-continuous correspondence from a non-empty, compact, convex set $Z \times W$ into itself such that for all $(z, w) \in Z \times W$, the set $F(z, w)$ is convex and non-empty. Convexity of $F(z, w)$ is of course immediate in the case of a singleton set. Suppose $(\tilde{z}, \tilde{w})=H(z, w, A)$ and $\left(\tilde{z}^{\prime}, \tilde{w}^{\prime}\right)=H\left(z, w, A^{\prime}\right)$ are two different elements of $F(z, w)$ and let $\alpha \in[0,1]$. By the definition it follows immediately that $\tilde{w}=\tilde{w}^{\prime}=\alpha \tilde{w}+(1-\alpha) \tilde{w}^{\prime}$. On the other hand,

$$
\alpha \tilde{z}_{i}+(1-\alpha) \tilde{z}_{i}^{\prime}=p_{i}+\frac{z_{i+1}}{\sum_{k} z_{k}}\left[\sum_{j} z_{j}\left(1-\left(\alpha A_{i+1 j}+(1-\alpha) A_{i+1, j}^{\prime}\right)\right)\right]
$$


We conclude that $\alpha H(z, w, A)+(1-\alpha) H\left(z, w, A^{\prime}\right)=H\left(z, w, \alpha A+(1-\alpha) A^{\prime}\right) \in G(z, w)$. Then applying Kakutani's fixed point theorem delivers the required result.

\section{Proof of Proposition 3}

We first show that $w_{i}$ is increasing in $i$. Obviously, $0=w_{0}<w_{1}$. Assume that $w_{0}<w_{1}<$ $\ldots<w_{i}$ for some $i \geq 1$. It is immediate that then $w_{i+1}>w_{i}$ because (by the induction step) $w_{i}>w_{i-1}$ and $\max \left\{\delta w_{i}, 1-\delta w_{j-1}\right\} \geq \max \left\{\delta w_{i-1}, 1-\delta w_{j-1}\right\}$ for all $j$.

To show that $w_{i}$ is concave in $i$, let $J=\left\{j: \delta w_{i}>1-\delta w_{j-1}\right\}$ and $J^{\prime}=\left\{j: \delta w_{i+1}>\right.$ $\left.1-\delta w_{j-1} \geq \delta w_{i}\right\}$. We then have

$$
\begin{aligned}
2\left(w_{i+2}-w_{i+1}\right) & =\delta\left(w_{i+1}-w_{i}\right)+\sum_{j \in J} q_{j} \delta\left(w_{i+1}-w_{i}\right)+\sum_{j \in J^{\prime}} q_{j}\left(\delta w_{i+1}-\left(1-\delta w_{j-1}\right)\right) \\
& \leq \delta\left(w_{i+1}-w_{i}\right)+\sum_{j} q_{j} \delta\left(w_{i+1}-w_{i}\right) \\
& =2 \delta\left(w_{i+1}-w_{i}\right) .
\end{aligned}
$$

\section{Proof of Corollary 4}

The first statement follows immediately from the fact that traders with deadline 1 face an outside option of $w_{0}=0$ in case of disagreement, together with the observation that $\delta w_{j-1}<1$, so that $1-\delta w_{j-1}>w_{0}$ and $A_{i 1}=1$.

(i) Let $A_{i j}<1$. Then $\delta\left(v_{i-1}+v_{j-1}\right) \geq 1$. Concavity implies that $\delta\left(v_{i}+v_{j-2}\right)>\delta\left(v_{i-1}+\right.$ $\left.v_{j-1}\right) \geq 1$ ), so that $A_{i+1, j-1}=0$.

(ii) Let $A_{i+1, j-1}>0$. Then by the previous result it follows that $A_{i j}=1$.

\section{Proof of Proposition 5}

Suppose there exists an equilibrium without delay when the inflow distribution is $p$. Let $\bar{p}_{1}$ be uniquely defined by $\bar{p}_{1}(\delta / 2)+\left(1-\bar{p}_{1}\right)(\delta / 2)^{N}=1-W A(p, \delta)$. Let $\bar{p}$ denote the extreme inflow distribution with fraction $\bar{p}_{1}$ of traders with deadline 1 and fraction $1-\bar{p}_{1}$ traders with deadline $N$. By definition of $\bar{p}_{1}$, this alternative inflow distribution satisfies $W A(\bar{p}, \delta)=W A(p, \delta)$ and thus must result in the same payoffs for all types as the original inflow distribution. On the other hand, when there are just two types and there is no delay, payoffs must satisfy the following equalities:

$$
\begin{gathered}
v_{1}=\bar{p}(1 / 2)+(1-\bar{p})(1 / 2)\left(1-\delta v_{N-1}\right)=\frac{1}{2}\left(1-(1-\bar{p}) \delta v_{N-1}\right) \\
v_{N}=(1-\bar{p})(1 / 2)+\bar{p}\left(\frac{1}{2} \delta v_{N-1}+\frac{1}{2}\right)=\frac{1}{2}\left(1+\bar{p} \delta v_{N-1}\right) .
\end{gathered}
$$

Using that no delay implies that $\delta v_{N-1} \leq 1 / 2$, it follows immediately that $v_{1}>1 / 4, v_{N-1}<3 / 4$, 
and $v_{N}<2 v_{1}$.

\section{Proof of Proposition 6}

There is no delay if and only if $2 \delta v_{N-1} \leq 1$, where $v_{N-1}$ is as defined in Proposition 5 .

\section{Proof of Corollary 8}

(i) Let $p^{\prime}$ first-order stochastically dominate $p$. Then $W A(p, \delta)<W A\left(p^{\prime}, \delta\right)$. Therefore

$$
\frac{1}{2 \delta} \geq \frac{1-\left(\frac{1}{2} \delta\right)^{N-1}}{2 W A(p, \delta)} \geq \frac{1-\left(\frac{1}{2} \delta\right)^{N-1}}{2 W A\left(p^{\prime}, \delta\right)}
$$

From Proposition 5 it follows that $v_{j}(p, \delta) / v_{j}\left(p^{\prime}, \delta\right)=W A\left(p^{\prime}, \delta\right) / W A(p, \delta)>1$.

(ii) Let $p^{\prime}$ second-order stochastically dominate $p$. Then $W A(p, \delta)<W A\left(p^{\prime}, \delta\right)$ and the statement follows from Propositions 5 and 6.

(iii) Let $L(\delta)=\delta-\left(\frac{1}{2}\right)^{N-1} \delta^{N}-\sum_{i=1}^{N} p_{i}\left(1-\left(\frac{1}{2} \delta\right)^{i}\right)$. We will show that $L(\delta)$ is increasing for $\delta<1$ which proves the claim as Proposition 6 states that there exists an equilibrium without delay if and only if $L(\delta) \leq 0$. Observe that for all natural numbers $N$ and any $\delta \in[0,1)$

$$
2^{N-1}-N \delta^{N-1}>2^{N-1}-N \geq 0
$$

so that

$$
L^{\prime}(\delta)=1-\frac{N \delta^{N-1}}{2^{N-1}}+\sum_{i=1}^{N} p_{i} \frac{i \delta^{i-1}}{2^{i}}>0 .
$$

Note that if $v_{1}\left(p, \delta^{\prime}\right) \leq v_{1}(p, \delta)$, then it would follow from $(2)$ that $v_{i}\left(p, \delta^{\prime}\right)<v_{i}(p, \delta)$ for all $i>1$. This is impossible because total surplus is equal to 1 in both cases. Hence, we conclude that $v_{1}\left(p, \delta^{\prime}\right)>v_{1}(p, \delta)$. In a similar fashion it can be shown that $v_{N}\left(p, \delta^{\prime}\right)<$ $v_{N}(p, \delta)$.

(iv) Suppose there is delay. Then $w_{N}>w_{N-1} \geq \delta w_{N-1} \geq 1 / 2$. Hence, every new trader entering the market (before learning his deadline) expects to obtain strictly more than $1 / 2$. This is not sustainable.

\section{Proof of Proposition 11}

Let $Z=\left\{z \in \Re_{+}^{N}: \sum_{i} z_{i} \geq 1\right.$ and $\left.z_{i} \leq N+1-i\right\}$ and let $W=\left\{w \in \Re_{+}^{N}: w_{1} \leq w_{2} \leq \ldots \leq\right.$ $\left.w_{N} \leq 1\right\}$. Let $\mathcal{M}$ denote the set of all $N \times N$ matrices with entries in the interval $[0,1]$ whose 
row sums are equal to 1 . For any $w \in W$, let $X(w)=\left\{\delta w_{0}, \delta w_{1}, \ldots, \delta w_{N-1}\right\}$ denote the set of (potentially optimal) offers. (Here $w_{0}=0$.) The entry $M_{i j}$ of the matrix $M$ is to be interpreted as the probability with which a proposer with deadline $i$ offers $\delta w_{j-1}$.

Consider the following correspondence $G: Z \times W \rightrightarrows Z \times W \times \mathcal{M}$ :

$G(z, w)=\left\{(z, w, M): M_{i j}=0\right.$ if $\delta w_{j-1} \notin \arg \max _{x \in X(w)}\left\{(1-x) \sum_{j: \delta w_{j-1} \leq x} z_{j}+\delta w_{i-1} \sum_{j: \delta w_{j-1}>x} z_{j}\right\}$.

The correspondence $G$ adds (mixed) strategies of proposers that are myopically optimal. That is, they are optimal given the distribution of deadlines implied by $z$, and under the further assumptions that a responder with deadline $j$ accepts proposal $x$ if and only if $x \geq \delta w_{j-1}$ and that rejected proposals yield proposer with deadline $i$ an expected payoff equal to $w_{i-1}$ one period later.

We furthermore define the following mapping $H: Z \times W \times \mathcal{M} \rightarrow Z \times W$ :

$$
H(z, w, M)=(\tilde{z}, \tilde{w})
$$

where $\tilde{z}_{N}=p_{N}$ and for $i<N$,

$$
\tilde{z}_{i}=p_{i}+\frac{z_{i+1}}{2 \sum_{j} z_{j}}\left(\left[\sum_{k} M_{i+1} k \sum_{j: \delta w_{j-1}>\delta w_{k-1}} z_{j}\right]+\left[\sum_{j}\left[z_{j} \sum_{k: \delta v_{i}>\delta w_{k-1}} M_{j k}\right]\right]\right)
$$

and, for all $i$,

$$
\begin{aligned}
\tilde{w}_{i}= & \frac{1}{2 \sum_{j} z_{j}}\left(\sum_{k}\left[M_{i k}\left(\left(1-\delta w_{k-1}\right) \sum_{j: \delta v_{j-1} \leq \delta w_{k-1}} z_{j}+\delta w_{i-1} \sum_{j: \delta v_{j-1}>\delta w_{k-1}} z_{j}\right)\right]\right) \\
& +\frac{1}{2 \sum_{j} z_{j}}\left(\sum_{j} z_{j}\left[\sum_{k} \max \left\{\delta v_{i-1}, \delta w_{k-1}\right\} M_{j k}\right]\right) .
\end{aligned}
$$

The mapping $H$ recalculates the mass of traders with different deadlines from the proposal strategies given by $M$ (and given the responders' strategies described before) and updates the expected payoff of traders. Note that $\tilde{z}_{i} \leq p_{i}+z_{i+1} \leq 1+N+1-(i+1)=N+1-i$ and that $\tilde{w}_{i} \leq \tilde{w}_{i+1} \leq 1$ when $w_{i-1} \leq w_{i}$ so that $H$ really maps into $Z \times W$.

We now combine $G$ and $H$ to construct a correspondence $F: Z \times W \rightrightarrows Z \times W$ as follows:

$$
F(z, w)=\{H(z, w, M):(z, w, M) \in G(z, w)\} .
$$

$F$ is an upper semi-continuous correspondence from a non-empty, compact, convex set $Z \times W$ 
into itself such that for all $(z, w) \in Z \times W$, the set $F(z, w)$ is convex and non-empty. Convexity of $F(z, w)$ is of course immediate in the case of a singleton set. Suppose $(\tilde{z}, \tilde{w})=H(z, w, M)$ and $\left(\tilde{z}^{\prime}, \tilde{w}^{\prime}\right)=H\left(z, w, M^{\prime}\right)$ are two different elements of $F(z, w)$ and let $\alpha \in[0,1]$. By the definition it follows immediately that $\left(z, w, \alpha M+(1-\alpha) M^{\prime}\right) \in G(z, w)$. Because of the linearity in $M$, it is straightforward that

$$
\alpha(\tilde{z}, \tilde{w})+(1-\alpha)\left(\tilde{z}^{\prime}, \tilde{w}^{\prime}\right)=H\left(z, w, \alpha M+(1-\alpha) M^{\prime}\right)
$$

Hence, $\alpha(\tilde{z}, \tilde{w})+(1-\alpha)\left(\tilde{z}^{\prime}, \tilde{w}^{\prime}\right) \in F(z, w)$ and applying Kakutani's fixed point theorem delivers the required result.

\section{Proof of Proposition 12}

First, assume the inequality is satisfied and consider the following strategies. All types offer as a proposer $\delta / 2$ to the opponent and keep $1-\delta / 2$ for themselves. Types with deadlines $i>1$ accept any proposal that yields them at least $\delta / 2$. Type 1 accepts any proposal that yields him a nonnegative payoff. It is clear that these strategies yield all traders an expected payoff of $1 / 2$. Also, given the proposer's strategies, it is optimal to accept any proposal equal to or above $\delta / 2$ and to reject (in the case of deadlines higher than 1) any lower proposals. For a trader with deadline 1 it is obviously optimal to accept any nonnegative offer. The only remaining question is whether some trader could do better by making a different proposal. Given the responder's strategies, the only alternative strategy that could possibly give a higher payoff would be to offer 0 (in the hope of being matched with a trader whose deadline is about to expire). Conditional on being a proposer (with deadline $i>1$ ) following the outlined strategy yields $1-\delta / 2$. Offering 0 will only be accepted by traders with deadline equal to 1 , so this yields an expected payoff of $p_{1} \times 1+\left(1-p_{1}\right) \times \delta v_{i-1}=\delta / 2+p_{1}(1-\delta / 2)$. The equilibrium condition is thus

$$
1-\delta / 2 \geq \delta / 2+p_{1}(1-\delta / 2)
$$

or, equivalently,

$$
p_{1} \leq 2(1-\delta) /(2-\delta)
$$

Second, suppose the inequality is not satisfied and suppose there exists an equilibrium with $v_{i}=v$ for all $i$. Clearly, $v \leq 1 / 2$. In such equilibrium offers above $\delta v$ must be accepted and offers below must be rejected (except for traders with deadline 1 who will accept any nonnegative offer). Therefore, the only offers that can possibly be made in equilibrium are $\delta v$ and 0 . If 0 is never offered then it follows that $v=1 / 2$ but then traders with high deadline are better off 
offering 0 , as we have seen before. Hence, some traders must propose 0 with positive probability. But that implies that $v_{i}<v_{i+1}$ for all $i$ as a trader with deadline $i+1$ can imitate a trader with deadline $i$ but has an extra chance to get a positive payoff in the event of being proposed $i$ times an offer of zero.

\section{Proof of Proposition 13}

Traders with deadline $j+1$ can always use the same strategy that traders with deadline $j$ use. Hence, $v_{j+1} \geq v_{j}$. From the previous proposition we know that not all traders receive the same payoff. Hence, for some deadline $k$ we have $v_{1} \leq \ldots \leq v_{k}<v_{k+1} \leq \ldots \leq v_{N}$. This implies that a trader with deadline $k$ cannot imitate the trader with deadline $k+1$. Hence, there must be a positive probability that a trader with deadline $k+1$ will still be in the market when his deadline has reduced to 1 . This in turn implies that for any trader with deadline $j<k+1$ there is a positive probability that he will remain in the market for $j$ periods. In particular, this is the case for a trader with deadline 2. Clearly, this means that delay occurs with positive probability.

First we show that $v_{1}<v_{2}$. This is obvious if the zero offer is made with positive probability, because then a trader with deadline 2 could just mimic the behavior of a trader with deadline 1, except for the case where a zero offer is received, which should be rejected. Similarly, if the trader with deadline 1 makes an offer in equilibrium which is rejected with positive probability, then the trader with deadline 2 can mimic a trader with deadline 1 . In case of being chosen as a proposer and the proposal being rejected, the trader with deadline 2 will have another chance to obtain a positive payoff. Hence, also in this case we must have $v_{1}<v_{2}$. So let us assume that the zero offer is not made and that traders with deadline 1 make a proposal that is accepted for sure. Then the lowest offer that can be made in equilibrium equals $\delta v_{1}$, which a trader with deadline 2 will accept with probability 1 in equilibrium. We know that a trader with deadline 2 will sometimes delay in equilibrium. This can only happen when he makes a proposal that is rejected by some trader(s), let us say, $\delta v_{m-1}<\delta v_{N-1}$. We can only have that $v_{1}=v_{2}$ if in fact trader 2, as a proposer, is indifferent between making the offers $\delta v_{m-1}$ and $\delta v_{N-1}$. But if this is the case, trader $k$ can mimic the behavior of a trader with deadline $k+1$ as long as the remaining deadline is strictly above 1 , and make the proposal that is certainly accepted when the deadline has reduced to 1 . In this way trader $k$ can obtain the same payoff as trader $k+1$, which is a contradiction. Hence, we have established that $v_{1}<v_{2}$.

It follows immediately that for all traders with $j<k$ that $v_{j}<v_{j+1}$. Namely, trader $j+1$ can mimic trader $j$ as long as the remaining deadline is above 2, and use the equilibrium strategy of a trader with deadline 2 when the deadline has reduced to 2 . In this way trader $j+1$ guarantees 
a payoff strictly higher than what trader $j$ can get.

We thus have either that (i) $v_{1}<v_{2}<\ldots<v_{N}$ or (ii) there exists a deadline $j>k$ such that $v_{1}<\ldots<v_{j}=v_{j+1} \leq \ldots \leq v_{N}$.

Suppose we are in case (ii). As argued before, it must be the case that there is a positive probability of delay for all traders with deadline $i$ such that $1<i<j+1$. Because the trader with deadline $j+1$ could imitate the trader with deadline $j$, but cannot do better than him, the zero offer is never made. (Namely, if the zero offer is made with positive probability, then he could do strictly better by not accepting the zero offer when his deadline has reduced to 2.) Consider now the possibility of trader $j+1$ imitating trader $j$ for one period. If a deal is concluded, he would obtain the same payoff as the trader with deadline $j$ in that circumstance. On the other hand, if there is delay (which happens with positive probability, then his future expected payoff is $v_{j}>v_{j-1}$. Hence, trader $j+1$ can guarantee a strictly higher payoff than $j$ and case (ii) cannot occur. We conclude that in any equilibrium $v_{1}<\ldots<v_{N}$.

Finally, it is straightforward to verify that deadlines are missed in equilibrium when, for example, $p=(0.99,0.01)$. In this case every proposer will offer zero to the responder, which is only accepted by traders with deadline 1 . Hence, with positive probability deadlines are missed.

Proof of Proposition 14. We already know that in an equilibrium without delay all traders make the same offer of $\delta / 2$. Consider an SSPE configuration without delay such that $v_{j-1}<v_{j}$ for all $j$. Denote $Q_{m}=q_{1}+\ldots+q_{m}$ for any $1 \leq m \leq N$. Then $Q_{m}<Q_{m+1}$ and $Q_{N}=1$.

$$
\begin{aligned}
\left(1-\delta v_{i}\right) Q_{i+1}+\delta v_{j-1}\left(1-Q_{i+1}\right) & \leq\left(1-\delta v_{i-k}\right) Q_{i-k+1}+\delta v_{j-1}\left(1-Q_{i-k+1}\right) \\
\Leftrightarrow Q_{i+1}\left(1-\delta v_{i}-\delta v_{j-1}\right) & \leq Q_{i-k+1}\left(1-\delta v_{i-k}-\delta v_{j-1}\right) \\
\Rightarrow Q_{i+1}\left(1-\delta v_{i}-\delta v_{j}\right) & <Q_{i-k+1}\left(1-\delta v_{i-k}-\delta v_{j}\right) \\
\Leftrightarrow\left(1-\delta v_{i}\right) Q_{i+1}+\delta v_{j}\left(1-Q_{i-k+1}\right) & <\left(1-\delta v_{i-k}\right) Q_{i-k+1}+\delta v_{j}\left(1-Q_{i-k+1}\right)
\end{aligned}
$$

The first inequality states that a trader with deadline $j$ weakly prefers to offer $\delta v_{i-k}$ rather than $\delta v_{i}$. The last inequality says that a trader with deadline $j+1$ strictly prefers to offer $\delta v_{i-k}$ rather than $\delta v_{i}$. 


\section{References}

Admati, A.R. and M. Perry (1987). "Strategic delay in bargaining," Review of Economic Studies, Vol. 54, pp. 345-364.

Anwar, A.W. and J. Sákovics (2007). "A decentralized market for a perishable good," The B.E. Journal of Theoretical Economics, Vol. 7, Iss. 1 (Contributions), Article 7.

Bester, H. and J. Sákovics (2001). "Delegated Bargaining and Renegotiation," Journal of Economic Behavior and Organization, Vol. 45, pp. 459-473.

Bose, G. (1996). "Bargaining Economies with Patient and Impatient Agents: Equilibria and Intermediation," Games and Economic Behavior, Vol. 14, pp. 149-172.

Burtraw, D. (1992). "Strategic Delegation in Bargaining," Economics Letters, Vol. 38, pp. 181-185.

Cai, H. (2000). "Bargaining on Behalf of a Constituency, Journal of Economic Theory, Vol. 92, pp. 234-273.

Chavez, A. and P. Maes (1996). "Kasbah: An Agent Marketplace for Buying and Selling Goods,", Proceedings of the First International Conference on the Practical Application of Intelligent Agents and Multi-Agent Technology, London, April 1996.

Damiano, E., Li, H. and W. Suen (2005). "Unravelling of Dynamic Sorting," Review of Economic Studies, Vol. 72, pp. 1057-1076

Duffie, D., N. Gârleanu, and L.H. Pedersen (2005). "Over-the-counter markets," Econometrica, Vol. 73, pp. 1815-1847.

Fershtman, C. and D. Seidmann (1993). "Deadline effects and inefficient delay in bargaining with endogenous commitment," Journal of Economic Theory, Vol. 60, pp. 306-321.

Gale, D. (1987). "Limit theorems for markets with sequential bargaining," Journal of Economic Theory, Vol. 43, pp. 20-54.

Gale, D. (2000). Strategic Foundations of General Equilibrium: Dynamic Matching and Bargaining Games. Cambridge and New York. Cambridge University Press.

Haller, H. and S. Holden (1990). "A letter to the editor on wage bargaining," Journal of Economic Theory, Vol. 52, pp. 232-236.

Hurkens, S. and N. Vulkan (2006). "Dynamic Matching and Bargaining: The Role of Deadlines." Barcelona GSE Research Network WP 188.

Jackson, M.O. and T.R. Palfrey (1998). "Efficiency and voluntary implementation in markets with repeated pairwise bargaining," Econometrica, Vol. 66, pp. 1353-1388.

Jennings N.R., P. Faratin, M.J. Johnson, T.J. Norman, P. O’Brien, and M.E. Wiegand (1996). "Agent-based business process management," International Journal of Cooperative In- 
formation Systems, Vol. 5, pp. 105-130.

Ma, C.A. and M. Manove (1993). "Bargaining with Deadlines and Imperfect Player Control," Econometrica, Vol. 61, pp. 1313-1339.

Manea, M. (2012). "Bargaining in Dynamic Markets with Multiple Populations." mimeo MIT.

Merlo, A. and F. Ortalo-Magné (2004). "Bargaining over residential real estate: evidence from England," Journal of Urban Economics, Vol. 56, pp. 192-216.

Merlo, A. and C. Wilson (1995). "A stochastic model of sequential bargaining with complete information," Econometrica, Vol. 63, pp. 371-399.

Mortensen, D.T. and R. Wright (2002). "Competitive pricing and efficiency in search equilibrium," International Economic Review, Vol. 43, pp. 1-20.

Moreno, D. and J. Wooders 82002). "Prices, delay, and the dynamics of trade," Journal of Economic Theory, Vol. 104, pp. 304-339.

Ponsati, C. (1995). "The Deadline Effect: A Theoretical Note," Economics Letters, Vol. 48, pp. 281-285.

Rubinstein, A. (1982). "Perfect Equilibrium in a Bargaining Model," Econometrica, Vol. 50, pp. $97-109$.

Rubinstein, A. and A. Wolinsky (1985). "Equilibrium in a market with sequential bargaining.," Econometrica, Vol. 53, pp. 1133-1150.

Sákovics, J. (1993). "Delay in bargaining games with complete information," Journal of Economic Theory, Vol. 59, pp. 78-95.

Samuelson, L. (1992). "Disagreement in Markets with Matching and Bargaining," Review of Economic Studies, Vol. 59, pp. 177-185.

Sandholm, T. and N. Vulkan (2000). "Bargaining with Deadlines,". Proceedings of the National Conference on Artificial Intelligence (AAAI), Orlando, FL.

Satterthwaite, M. and A. Shneyerov (2007). "Dynamic Matching, Two-sided Incomplete Information, and Participation Costs: Existence and Convergence to perfect competition," Econometrica, Vol. 75, pp. 155-200.

Segendorff, B. (1998). "Delegation and Threat in Bargaining," Games and Economic Behavior, Vol. 23, pp. 266-283.

Serrano, R. (2002). "Decentralized Information and and the Walrasian Outcome: A Pairwise Meetings Market with Private values," Journal of Mathematical Economics, Vol. 38 , pp. 65-89.

Shimer, R. and L. Smith (2000). "Assortative Matching and Search," Econometrica, Vol. 68, pp. 343-369. 
Shneyerov, A. and A. Wong (2010a). "The Rate of Convergence to Perfect Competition of Matching and Bargaining Mechanisms," Journal of Economic Theory, Vol. 145, pp. 1164-1187. Shneyerov, A. and A. Wong (2010b). "Bilateral Matching and Bargaining with Private Information," Games and Economic Behavior, Vol. 68, pp. 748-762.

Sobel, J. and I. Takahashi (1983). "A multistage model of bargaining," Review of Economic Studies, Vol. 50, pp. 411-426.

Ståhl, I. (1972). Bargaining Theory. Stockholm School of Economics, Stockholm.

Vayanos, D. and T. Wang (2007). "Search and endogenous concentration of liquidity in asset markets," Journal of Economic Theory, Vol. 136, pp. 66-104.

Yildiz, M. (2004). "Optimism, Deadline Effect, and Stochastic Deadlines," mimeo MIT. 